\title{
Existence and Uniqueness of Solutions to Third-Order Boundary Value Problems
}

\author{
S. S. ALMUTHAYBIRI ${ }^{1,2}$, J. M. JONNALAGADDA ${ }^{3}$ and \\ C. C. TISDELL ${ }^{4 *}$
}

Received on October 27, 2020 / Accepted on January 7, 2021

\begin{abstract}
The purpose of this research is to connect fixed point methods with certain third-order boundary value problems in new and interesting ways. Our strategy involves an analysis of the problem under consideration within closed and bounded sets. We develop sufficient conditions under which the associated mappings will be contractive and invariant in these sets, which generates new advances concerning the existence, uniqueness and approximation of solutions.
\end{abstract}

Keywords: third-order boundary value problem, existence, uniqueness and approximation of solutions, contraction mappings, fixed point theorems, closed and bounded sets, two and three-point boundary conditions.

\section{INTRODUCTION}

Consider the following boundary value problem (BVP):

$$
\begin{array}{r}
x^{\prime \prime \prime}+f\left(t, x, x^{\prime}, x^{\prime \prime}\right)=0, \quad t \in[a, b] ; \\
x(a)=0, x^{\prime}(a)=0, x^{\prime}(b)-\theta x^{\prime}(\eta)=\lambda .
\end{array}
$$

Above: $f$ is a real-valued continuous function; $a<\eta<b ;(b-a)-\theta(\eta-a) \neq 0$; and $\lambda \in \mathbb{R}$. We define a solution to (1.1), (1.2) to be a real-valued function $x=x(t)$ that has a third-order derivative that is continuous on $[a, b]$ (which we denote by $x \in C^{3}([a, b])$; and $x$ satisfies (1.1) on $[a, b]$ and the boundary conditions (1.2). Observe that if $\theta \neq 0$ then (1.2) contains information involving three points; while if $\theta=0$ then (1.2) expresses data at two points.

\footnotetext{
*Corresponding author: Christopher C. Tisdell - E-mail: cct@unsw.edu.au

${ }^{1}$ School of Mathematics, The University of New South Wales, Sydney, New South Wales 2052, Australia

2 Department of Mathematics, College of Science and Arts in Uglat Asugour, Qassim University, Buraydah, Kingdom of Saudi Arabia - E-mail: s.almuthaybiri@qu.edu.sa https://orcid.org/0000-0002-9399-3253

${ }^{3}$ Department of Mathematics, Birla Institute of Technology and Science Pilani, Hyderabad, 500078, Telangana, India E-mail: j.jaganmohan@hotmail.com https://orcid.org/0000-0002-1310-8323

${ }^{4}$ School of Mathematics, The University of New South Wales, Sydney, New South Wales 2052, Australia - E-mail: cct@unsw.edu.au https://orcid.org/0000-0002-3387-2505
} 
There are a number of special cases of (1.1) that have been of interest, especially in the modelling of fluid flow. For example, in the context of mixed-convection boundary-layer flow in a saturated porous medium, the form

$$
x^{\prime \prime \prime}+x x^{\prime \prime}=0
$$

was derived in [6,7] from the equations of motion. Here $x$ is an unknown part of a stream function that is derived from the governing equations and is to be determined.

Another special case of (1.1) is the classic form

$$
x^{\prime \prime \prime}-\left(x^{\prime}\right)^{2}+x x^{\prime \prime}-1=0
$$

which arises in the study of laminar flow against an infinite plate, see [9, pp.232-233]. Roughly speaking, in the above notation, the function $x$ depends on distance and is part of a stream function that is related to the velocity field of a fluid experiencing two-dimensional laminar flow against an infinite plate, where a stagnation point is present.

One of the challenges regarding the mathematical analysis of equations (1.3) and (1.4) is the fact that they do not satisfy a Lipschitz condition on unbounded sets and thus they do not satisfy the conditions that are associated with Lipschitz-type existence and uniqueness theorems in unbounded domains.

The right-hand boundary conditions in (1.2) are interesting, for example, due to their ability to approximate important forms. Firstly, if $\eta$ is fixed and close to $b$ with $\theta=1$ and $\lambda=b-\eta$, then the three-point boundary condition may be considered as approximating the boundary condition

$$
x^{\prime \prime}(b)=1 \text {. }
$$

Secondly, if $a=0, \theta=0$ and $\lambda=1$ then for large values of $b>0$ the boundary conditions (1.2) can be thought of as forming an approximation to the conditions that arise in the modelling associated with (1.3) and (1.4), namely

$$
x(0)=0, x^{\prime}(0)=0, x^{\prime}(\infty)=1
$$

The literature on third-order BVPs is significant in volume and scope and thus a full review is beyond the focus of this article, however, recent works regarding the existence, uniqueness and approximation of solutions to third-order BVPs that are connected with the ideas in our present article are $[2,11]$. Therein, a priori bounds were developed for the associated Green's function and then applied to ensure the BVP

$$
\begin{array}{r}
x^{\prime \prime \prime}+f(t, x)=0, \quad t \in[a, b] ; \\
x(a)=0, x^{\prime}(a)=0, x(b)=\theta x(\eta)
\end{array}
$$

admitted a unique solution via contractive maps.

Our particular interest in the existence, uniqueness and approximation of solutions to (1.1), (1.2) is motivated by the recent work in [5]. Therein, the authors skillfully developed a set of existence 
results for positive solutions to (1.1), (1.2) by drawing on ideas from fixed point theory, such as Krasnoselskii's Theorem and the Leray-Schauder Alternative. A numerical method was also introduced in [5] that employed Picard iterations [12].

The aim of the present work is to advance the current state of knowledge on (1.1), (1.2) in a way that complements and extends the work in $[2,5,11]$. A key strategy involves restricting our mathematical analysis to within closed and bounded sets, successfully navigating the aforementioned challenge of global Lipschitz-ness in the process.

We introduce some notation and other componentry associated with our work in Section 2. In Section 3 we establish new estimates on the integrals of derivatives of various Green's functions, including "sharp" estimates. These new estimates are applied to (1.1), (1.2) in Section 4 via fixed point theorems to ensure the existence and uniqueness of solutions under sufficient conditions. In addition, we establish some constructive results regarding the approximation of solutions through the use of Picard iterations.

\section{NOTATION AND OTHER COMPONENTRY}

The following two results on fixed points of contractive operators will form important components of our methods.

Theorem 1 (Banach, [3]). Let $X$ be a nonempty set and let $d$ be a metric on $X$ such that $(X, d)$ forms a complete metric space. If the mapping $T: X \rightarrow X$ satisfies

$$
d(T x, T y) \leq \alpha d(x, y), \text { for some } 0<\alpha<1 \text { and all } x, y \in X
$$

then there is a unique $z \in X$ such that $T z=z$.

Theorem 2 (Rus, [10]). Let $X$ be a nonempty set and let $d$ and $\delta$ be two metrics on $X$ such that $(X, d)$ forms a complete metric space. If the mapping $T: X \rightarrow X$ is continuous with respect to $d$ on $X$ and:

$$
\begin{array}{ll}
d(T x, T y) \leq c \delta(x, y), & \text { for some } c>0 \text { and all } x, y \in X \\
\delta(T x, T y) \leq \alpha \delta(x, y), & \text { for some } 0<\alpha<1 \text { and all } x, y \in X
\end{array}
$$

then there is a unique $z \in X$ such that $T z=z$.

For a recent discussion and comparison of the above theorems, see [2].

Herein, we will be concerned with the following notation, sets and metrics. Denote the set of realvalued functions that are continuous on $[a, b]$ by $C([a, b])$, and for $x, y \in C([a, b])$ and consider the following metrics:

$$
\begin{aligned}
d_{\infty}(x, y) & :=\max _{t \in[a, b]}|x(t)-y(t)| \\
\delta_{p}(x, y) & :=\left(\int_{a}^{b}|x(t)-y(t)|^{p} d t\right)^{1 / p}, \quad p>1 .
\end{aligned}
$$


Now consider the set of real-valued functions that are defined on $[a, b]$ that possess secondorder derivatives that are continuous therein. We denote this space by $C^{2}([a, b])$. For functions $x, y \in C^{2}([a, b])$ we furnish the following metrics from $d_{\infty}$ and $\delta_{p}$ :

$$
\begin{aligned}
d(x, y) & :=\max \left\{W_{0} d_{\infty}(x, y), W_{1} d_{\infty}\left(x^{\prime}, y^{\prime}\right), W_{2} d_{\infty}\left(x^{\prime \prime}, y^{\prime \prime}\right)\right\} ; \\
\delta(x, y) & :=L_{0} \delta_{p}(x, y)+L_{1} \delta_{p}\left(x^{\prime}, y^{\prime}\right)+L_{2} \delta_{p}\left(x^{\prime \prime}, y^{\prime \prime}\right) .
\end{aligned}
$$

The non-negative constants $W_{i}$ and $L_{i}$ will be appropriately defined in the statements or proofs of our main results.

\section{ESTABLISHING ESTIMATES: INTEGRALS OF GREEN'S FUNCTIONS}

In this section we establish a range of estimates for integrals involving various Green's functions from the BVP (1.1), (1.2). These results are of interest in their own right, and we will rely on them to help establish our existence, uniqueness and approximation theorems for solutions to (1.1), (1.2).

By employing the procedure in [5], it can be shown that the BVP (1.1), (1.2) can be equivalently reformulated as the integral equation

$$
\begin{aligned}
x(t)= & \frac{\lambda(t-a)^{2}}{2((b-a)-\theta(\eta-a))}+\int_{a}^{b} G(t, s) f\left(s, x(s), x^{\prime}(s), x^{\prime \prime}(s)\right) d s \\
& +\frac{\theta \lambda(t-a)^{2}}{2((b-a)-\theta(\eta-a))} \int_{a}^{b} G_{t}(\eta, s) f\left(s, x(s), x^{\prime}(s), x^{\prime \prime}(s)\right) d s, \quad t \in[a, b],
\end{aligned}
$$

where

$$
G(t, s)=\frac{1}{2(b-a)} \begin{cases}(s-a)\left((2 t-s) b-t^{2}-a(b-s)\right), & \text { for } a \leq s \leq t \leq b ; \\ (b-s)(t-a)^{2}, & \text { for } a \leq t \leq s \leq b,\end{cases}
$$

and so the partial derivative of $G(t, s)$ with respect to $t$ evaluated at $t=\eta$ is

$$
G_{t}(\eta, s)=\frac{1}{(b-a)} \begin{cases}(s-a)(b-\eta), & \text { for } a \leq s \leq \eta \leq b ; \\ (b-s)(\eta-a), & \text { for } a \leq \eta \leq s \leq b .\end{cases}
$$

We observe the following properties of the Green's function $G(t, s)$ defined in (3.2).

Theorem 1. The function $G(t, s)$ in (3.2) satisfies $G \geq 0$ on $[a, b] \times[a, b]$.

Proof. The case $a \leq t \leq s \leq b$ is straightforward due the product of non-negative functions therein.

We prove the case $a \leq s \leq t \leq b$ via simple inequalities. If we expand the numerator of $G$ and complete the square in $t$ then we obtain

$$
(s-a)\left((2 t-s) b-t^{2}-a(b-s)\right)=(s-a)\left((b-a)(b-s)-(b-t)^{2}\right) \geq 0,
$$

since $a \leq s \leq t \leq b$. 
Let us now develop a bound on the integral of $G$.

Theorem 2. The function $G(t, s)$ in (3.2) satisfies

$$
\int_{a}^{b} G(t, s) d s \leq \frac{(b-a)^{3}}{12}, \text { for all } t \in[a, b] .
$$

Inequality (3.4) is sharp in the sense that it is the best inequality possible.

Proof. For all $t \in[a, b]$ we have

$$
\begin{aligned}
\int_{a}^{b} G(t, s) d s & =\int_{a}^{t} G(t, s) d s+\int_{t}^{b} G(t, s) d s \\
& =\int_{a}^{t} \frac{(s-a)\left((2 t-s) b-t^{2}-a(b-s)\right)}{2(b-a)} d s+\int_{t}^{b} \frac{(b-s)(t-a)^{2}}{2(b-a)} d s \\
& =\frac{(t-a)^{3}(4 b-3 t-a)}{12(b-a)}+\frac{(t-a)^{2}(b-t)^{2}}{4(b-a)} \\
& =\frac{(3 b-2 t-a)(t-a)^{2}}{12} .
\end{aligned}
$$

Now, an application of basic calculus shows that the above expression achieves its maximum value on $[a, b]$ at $t=b$ which gives

$$
\max _{t \in[a, b]} \int_{a}^{b} G(t, s) d s=\frac{(b-a)^{3}}{12} .
$$

Let us now develop a bound related to the integral of $G_{t}$.

Theorem 3. The function $G(t, s)$ in (3.2) satisfies $G_{t} \geq 0$ for all $[a, b] \times[a, b]$ and

$$
\int_{a}^{b} G_{t}(t, s) d s \leq \frac{(b-a)^{2}}{8}, \text { for all } t \in[a, b] .
$$

Inequality (3.5) is sharp in the sense that it is the best inequality possible.

Proof. The non-negativity of $G_{t}$ follows immediately from its definition because $G_{t}$ involves the product of non-negative functions.

For all $t \in[a, b]$ we have

$$
\begin{aligned}
\int_{a}^{b} G_{t}(t, s) d s & =\int_{a}^{t} G_{t}(t, s) d s+\int_{t}^{b} G_{t}(t, s) d s \\
& =\int_{a}^{t} \frac{(s-a)(b-t)}{(b-a)} d s+\int_{t}^{b} \frac{(b-s)(t-a)}{(b-a)} d s \\
& =\frac{(b-t)(t-a)^{2}}{2(b-a)}+\frac{(t-a)(b-t)^{2}}{2(b-a)} \\
& =\frac{(b-t)(t-a)}{2} .
\end{aligned}
$$


An alternative to the above approach is to swap integration and differentiation to obtain

$$
\begin{aligned}
\int_{a}^{b} G_{t}(t, s) d s & =\frac{d}{d t} \int_{a}^{b} G(t, s) d s \\
& =\frac{d}{d t} \frac{(3 b-2 t-a)(t-a)^{2}}{12} \\
& =\frac{1}{2}(b-t)(t-a) .
\end{aligned}
$$

Now, basic calculus reveals that the above expression achieves its maximum value on $[a, b]$ at $t=(a+b) / 2$ which gives

$$
\max _{t \in[a, b]} \int_{a}^{b} G_{t}(t, s) d s=\frac{(b-a)^{2}}{8} .
$$

Let us now develop a bound on the integral of $G_{t}(\eta, s)$.

Theorem 4. The function $G_{t}(\eta, s)$ in (3.3) satisfies

$$
\int_{a}^{b} G_{t}(\eta, s) d s=\frac{1}{2}(b-\eta)(\eta-a) .
$$

Proof. We have

$$
\begin{aligned}
\int_{a}^{b} G_{t}(\eta, s) d s & =\int_{a}^{\eta} G_{t}(\eta, s) d s+\int_{\eta}^{b} G_{t}(\eta, s) d s \\
& =\int_{a}^{\eta} \frac{(s-a)(b-\eta)}{(b-a)} d s+\int_{\eta}^{b} \frac{(b-s)(\eta-a)}{(b-a)} d s \\
& =\frac{(b-\eta)(\eta-a)^{2}}{2(b-a)}+\frac{(\eta-a)(b-\eta)^{2}}{2(b-a)} \\
& =\frac{1}{2}(b-\eta)(\eta-a) .
\end{aligned}
$$

Thus we have constructed (3.6).

Let us now develop a bound related to the integral of $G_{t t}$.

Theorem 5. The function $G(t, s)$ in (3.2) satisfies

$$
\int_{a}^{b}\left|G_{t t}(t, s)\right| d s \leq \frac{(b-a)}{2}, \text { for all } t \in[a, b]
$$

Inequality (3.7) is sharp in the sense that it is the best inequality possible. 
Proof. For all $t \in[a, b]$ we have

$$
\begin{aligned}
\int_{a}^{b}\left|G_{t t}(t, s)\right| d s & =\int_{a}^{t} \frac{|-(s-a)|}{(b-a)} d s+\int_{t}^{b} \frac{|b-s|}{(b-a)} d s \\
& =\int_{a}^{t} \frac{s-a}{b-a} d s+\int_{t}^{b} \frac{b-s}{b-a} d s \\
& =\frac{(t-a)^{2}}{2(b-a)}+\frac{(b-t)^{2}}{2(b-a)} .
\end{aligned}
$$

Once again, this is non-negative, concave up function and its maximum value on $[a, b]$ is achieved at the end points of $[a, b]$. Its value at $t=a$ and $t=b$ are both $(b-a) / 2$ and thus we have established (3.7).

Combining the aforementioned theorems, we have the following results on bounds that will be particularly helpful in the sections that follow.

Theorem 6. For all $t \in[a, b]$ we have

$$
\begin{aligned}
& \int_{a}^{b} G(t, s) d s+\frac{|\theta||\lambda|(t-a)^{2}}{2|(b-a)-\theta(\eta-a)|} \int_{a}^{b} G_{t}(\eta, s) d s \\
& \leq \frac{(b-a)^{3}}{12}+\frac{|\theta||\lambda|(b-a)^{2}}{4|(b-a)-\theta(\eta-a)|}(b-\eta)(\eta-a) .
\end{aligned}
$$

Proof. The inequality follows from Theorem 2 and Theorem 4.

Theorem 7. For all $t \in[a, b]$ we have

$$
\begin{aligned}
& \int_{a}^{b} G_{t}(t, s) d s+\frac{|\theta||\lambda|(t-a)}{|(b-a)-\theta(\eta-a)|} \int_{a}^{b} G_{t}(\eta, s) d s \\
& \leq \frac{(b-a)^{2}}{8}+\frac{|\theta||\lambda|(b-a)}{2|(b-a)-\theta(\eta-a)|}(b-\eta)(\eta-a) .
\end{aligned}
$$

Proof. The inequality follows from Theorem 3 and Theorem 4.

Theorem 8. For all $t \in[a, b]$ we have

$$
\begin{gathered}
\int_{a}^{b} G_{t t}(t, s) d s+\frac{|\theta||\lambda|}{|(b-a)-\theta(\eta-a)|} \int_{a}^{b} G_{t}(\eta, s) d s \\
\quad \leq \frac{b-a}{2}+\frac{|\theta||\lambda|}{2|(b-a)-\theta(\eta-a)|}(b-\eta)(\eta-a) .
\end{gathered}
$$

Proof. The inequality follows from Theorem 4 and Theorem 5. 


\section{EXISTENCE, UNIQUENESS AND APPROXIMATION}

We now construct a range of results that guarantee the existence, uniqueness and approximation of solutions to (1.1), (1.2). Our strategy employs an analysis within closed and bounded sets, the metrics in Section 2, the bounds formed in Section 3, and through fixed point approaches.

So that we do not rely on long and complicated expressions, we define the following constants to simplify our bounds from Section 3. The following notation will be used in the statement and proof of our main results:

$$
\begin{aligned}
& \beta_{0}:=\frac{(b-a)^{3}}{12}+\frac{|\theta||\lambda|(b-a)^{2}}{4|(b-a)-\theta(\eta-a)|}(b-\eta)(\eta-a) \\
& \beta_{1}:=\frac{(b-a)^{2}}{8}+\frac{|\theta||\lambda|(b-a)}{2|(b-a)-\theta(\eta-a)|}(b-\eta)(\eta-a) \\
& \beta_{2}:=\frac{\mid \theta-a}{2}+\frac{|\theta||\lambda|}{2|(b-a)-\theta(\eta-a)|}(b-\eta)(\eta-a) .
\end{aligned}
$$

\subsection{Banach Fixed Point Approach}

We can now draw on the results of Sections 2 and 3 to generate new results concerning the existence, uniqueness and approximation of solutions to the BVP (1.1), (1.2) via Banach's fixed point theorem.

Theorem 1. Let $f: B \rightarrow \mathbb{R}$ be continuous and uniformly bounded by $M>0$ on the "block"

$$
\begin{gathered}
B:=\left\{(t, u, v, w) \in \mathbb{R}^{4}: t \in[a, b],\left|u-\frac{\lambda(t-a)^{2}}{2((b-a)-\theta(\eta-a))}\right| \leq R,\right. \\
\left.\left|v-\frac{\lambda(t-a)}{(b-a)-\theta(\eta-a)}\right| \leq \frac{\beta_{1}}{\beta_{0}} R,\left|w-\frac{\lambda}{(b-a)-\theta(\eta-a)}\right| \leq \frac{\beta_{2}}{\beta_{0}} R\right\},
\end{gathered}
$$

where $R>0$ is a constant and each $\beta_{i}$ is defined in (4.1) with $M \beta_{0} \leq R$. For $i=0,1,2$, let $L_{i}$ be non-negative constants (not all zero) such that

$$
\begin{array}{r}
\left|f\left(t, u_{0}, u_{1}, u_{2}\right)-f\left(t, v_{0}, v_{1}, v_{2}\right)\right| \leq \sum_{i=0}^{2} L_{i}\left|u_{i}-v_{i}\right|, \\
\text { for all }\left(t, u_{0}, u_{1}, u_{2}\right),\left(t, v_{0}, v_{1}, v_{2}\right) \in B .
\end{array}
$$

If

$$
L_{0} \beta_{0}+L_{1} \beta_{1}+L_{2} \beta_{2}<1
$$

then the BVP (1.1), (1.2) has a unique solution in $C^{3}([a, b])$ such that

$$
\left(t, x(t), x^{\prime}(t), x^{\prime \prime}(t)\right) \in B, \text { for all } t \in[a, b] .
$$


Proof. Consider the pair $\left(C^{2}([a, b]), d\right)$, where the constants $W_{i}$ in our $d$ in (2.4) are chosen to form

$$
d(x, y):=\max \left\{d_{\infty}(x, y), \frac{\beta_{0}}{\beta_{1}} d_{\infty}\left(x^{\prime}, y^{\prime}\right), \frac{\beta_{0}}{\beta_{2}} d_{\infty}\left(x^{\prime \prime}, y^{\prime \prime}\right)\right\},
$$

(that is, $W_{0}=1, W_{1}=\beta_{0} / \beta_{1}$ and $W_{2}=\beta_{0} / \beta_{2}$ ). Our pair forms a complete metric space. Now, for the constant $R>0$ in the definition of $B$, consider the following ball $\mathscr{B}_{R} \subset C^{2}([a, b])$ defined via

$$
\mathscr{B}_{R}:=\left\{x \in C^{2}([a, b]): d(x, \phi) \leq R\right\}
$$

where

$$
\phi(t):=\frac{\lambda(t-a)^{2}}{2((b-a)-\theta(\eta-a))} .
$$

Since $\mathscr{B}_{R}$ is a closed subspace of $C^{2}([a, b])$, the pair $\left(\mathscr{B}_{R}, d\right)$ forms a complete metric space.

Consider the operator $T: \mathscr{B}_{R} \rightarrow C^{2}([a, b])$ defined by

$$
\begin{aligned}
& (T x)(t):=\frac{\lambda(t-a)^{2}}{2((b-a)-\theta(\eta-a))} \\
& +\int_{a}^{b}\left[G(t, s)+\frac{\theta \lambda(t-a)^{2}}{2((b-a)-\theta(\eta-a))} G_{t}(\eta, s)\right] f\left(s, x(s), x^{\prime}(s), x^{\prime \prime}(s)\right) d s, t \in[a, b] .
\end{aligned}
$$

In view of (3.1) we wish to show that there exists a unique $x \in \mathscr{B}_{R}$ such that

$$
T x=x .
$$

Every such solution will also lie in $C^{3}([a, b])$ as can be directly shown by differentiating (3.1) and confirming the continuity.

To establish the existence and uniqueness to $T x=x$, we show that the conditions of Theorem 1 hold with $X=\mathscr{B}_{R}$.

Let us show $T: \mathscr{B}_{R} \rightarrow \mathscr{B}_{R}$. For $x \in \mathscr{B}_{R}$ and $t \in[a, b]$, consider

$$
\begin{aligned}
& |(T x)(t)-\phi(t)| \\
\leq & \int_{a}^{b}\left[G(t, s)+\frac{|\theta||\lambda|(t-a)^{2}}{2|(b-a)-\theta(\eta-a)|} G_{t}(\eta, s)\right]\left|f\left(s, x(s), x^{\prime}(s), x^{\prime \prime}(s)\right)\right| d s \\
\leq & M \beta_{0},
\end{aligned}
$$

where we have applied Theorem 6. Thus we have

$$
d_{\infty}(T x, \phi) \leq M \beta_{0} .
$$

Similarly,

$$
\begin{aligned}
& \left|(T x)^{\prime}(t)-\phi^{\prime}(t)\right| \\
\leq & \int_{a}^{b}\left[G_{t}(t, s)+\frac{|\theta||\lambda|(t-a)}{|(b-a)-\theta(\eta-a)|} G_{t}(\eta, s)\right]\left|f\left(s, x(s), x^{\prime}(s), x^{\prime \prime}(s)\right)\right| d s \\
\leq & M \beta_{1}
\end{aligned}
$$


where we have applied Theorem 7. Then, we have

$$
d_{\infty}\left((T x)^{\prime}, \phi^{\prime}\right) \leq M \beta_{1} .
$$

In addition, via similar arguments, we obtain

$$
\left|(T x)^{\prime \prime}(t)-\phi^{\prime \prime}(t)\right| \leq M \beta_{2},
$$

by drawing on Theorem 8 . Consequently, we have

$$
d_{\infty}\left((T x)^{\prime \prime}, \phi^{\prime \prime}\right) \leq M \beta_{2}
$$

Thus, for all $x \in \mathscr{B}_{R}$ we have

$$
\begin{aligned}
d(T x, \phi) & =\max \left\{d_{\infty}(T x, \phi), \frac{\beta_{0}}{\beta_{1}} d_{\infty}\left((T x)^{\prime}, \phi^{\prime}\right), \frac{\beta_{0}}{\beta_{2}} d_{\infty}\left((T x)^{\prime \prime}, \phi^{\prime \prime}\right)\right\} \\
& \leq \max \left\{M \beta_{0}, \frac{\beta_{0}}{\beta_{1}} M \beta_{1}, \frac{\beta_{0}}{\beta_{2}} M \beta_{2}\right\} \\
& =\max \left\{M \beta_{0}, M \beta_{0}, M \beta_{0}\right\} \\
& =M \beta_{0} \\
& \leq R
\end{aligned}
$$

where the final inequality holds by assumption. Thus, for all $x \in \mathscr{B}_{R}$ we have $T x \in \mathscr{B}_{R}$ so that $T: \mathscr{B}_{R} \rightarrow \mathscr{B}_{R}$.

Let us now show that $T$ is contractive on $\mathscr{B}_{R}$ with respect to $d$. For $x, y \in \mathscr{B}_{R}$ and $t \in[a, b]$, consider

$$
\begin{aligned}
& |(T x)(t)-(T y)(t)| \\
\leq & \int_{a}^{b} G(t, s)\left|f\left(s, x(s), x^{\prime}(s), x^{\prime \prime}(s)\right)-f\left(s, y(s), y^{\prime}(s), y^{\prime \prime}(s)\right)\right| \\
+ & \frac{|\theta||\lambda|(t-a)^{2}}{2|(b-a)-\theta(\eta-a)|} G_{t}(\eta, s)\left|f\left(s, x(s), x^{\prime}(s), x^{\prime \prime}(s)\right)-f\left(s, y(s), y^{\prime}(s), y^{\prime \prime}(s)\right)\right| d s \\
\leq & \int_{a}^{b}\left[G(t, s)+\frac{|\theta||\lambda|(t-a)^{2}}{2|(b-a)-\theta(\eta-a)|} G_{t}(\eta, s)\right]\left(\sum_{i=0}^{2} L_{i}\left|x^{(i)}(s)-y^{(i)}(s)\right|\right) d s \\
\leq & \beta_{0}\left(L_{0} d_{\infty}(x, y)+L_{1} d_{\infty}\left(x^{\prime}, y^{\prime}\right)+L_{2} d_{\infty}\left(x^{\prime \prime}, y^{\prime \prime}\right)\right) \\
\leq & \beta_{0}\left(L_{0} d(x, y)+L_{1} \frac{\beta_{1}}{\beta_{0}} d(x, y)+L_{2} \frac{\beta_{2}}{\beta_{0}} d(x, y)\right) \\
= & \left(L_{0} \beta_{0}+L_{1} \beta_{1}+L_{2} \beta_{2}\right) d(x, y),
\end{aligned}
$$

where we have applied (3.4) and (4.2).

Similarly, we can show

$$
\begin{aligned}
\left|(T x)^{\prime}(t)-(T y)^{\prime}(t)\right| & \leq \beta_{1}\left(L_{0}+L_{1} \frac{\beta_{1}}{\beta_{0}}+L_{2} \frac{\beta_{2}}{\beta_{0}}\right) d(x, y) \\
\left|(T x)^{\prime \prime}(t)-(T y)^{\prime \prime}(t)\right| & \leq \beta_{2}\left(L_{0}+L_{1} \frac{\beta_{1}}{\beta_{0}}+L_{2} \frac{\beta_{2}}{\beta_{0}}\right) d(x, y) .
\end{aligned}
$$


Thus, for all $x, y \in \mathscr{B}_{R}$ we have

$$
\begin{aligned}
& d(T x, T y) \\
= & \max \left\{d_{\infty}(T x, T y), \frac{\beta_{0}}{\beta_{1}} d_{\infty}\left((T x)^{\prime},(T y)^{\prime}\right), \frac{\beta_{0}}{\beta_{2}} d_{\infty}\left((T x)^{\prime \prime},(T y)^{\prime \prime}\right)\right\} \\
\leq & \left(L_{0} \beta_{0}+L_{1} \beta_{1}+L_{2} \beta_{2}\right) d(x, y) .
\end{aligned}
$$

Due to our assumption (4.3) we see that $T$ is a contractive map on $\mathscr{B}_{R}$. Thus all of the conditions of Theorem 1 hold with $X=\mathscr{B}_{R}$. We conclude that the operator $T$ has a unique fixed point in $\mathscr{B}_{R} \subset C^{2}([a, b])$. This solution is also in $C^{3}([a, b])$ and we have equivalently shown that the BVP (1.1), (1.2) has a unique solution.

The following varitation of Theorem 1 is now established.

Theorem 2. Let $f: C \rightarrow \mathbb{R}$ be continuous and uniformly bounded by $M>0$ on the "block"

$$
\begin{array}{r}
C:=\left\{(t, u, v, w) \in \mathbb{R}^{4}: t \in[a, b],\left|u-\frac{\lambda(t-a)^{2}}{2((b-a)-\theta(\eta-a))}\right| \leq \frac{\beta_{0}}{\beta_{1}} R,\right. \\
\left.\left|v-\frac{\lambda(t-a)}{(b-a)-\theta(\eta-a)}\right| \leq R,\left|w-\frac{\lambda}{(b-a)-\theta(\eta-a)}\right| \leq \frac{\beta_{2}}{\beta_{1}} R\right\},
\end{array}
$$

where $R>0$ is a constant and each $\beta_{i}$ is defined in (4.1) with $M \beta_{1} \leq R$. For $i=0,1,2$, let $L_{i}$ be non-negative constants (not all zero) such that

$$
\begin{array}{r}
\left|f\left(t, u_{0}, u_{1}, u_{2}\right)-f\left(t, v_{0}, v_{1}, v_{2}\right)\right| \leq \sum_{i=0}^{2} L_{i}\left|u_{i}-v_{i}\right|, \\
\text { for all }\left(t, u_{0}, u_{1}, u_{2}\right),\left(t, v_{0}, v_{1}, v_{2}\right) \in C .
\end{array}
$$

If

$$
L_{0} \beta_{0}+L_{1} \beta_{1}+L_{2} \beta_{2}<1
$$

then the BVP (1.1), (1.2) has a unique solution in $C^{3}([a, b])$ such that

$$
\left(t, x(t), x^{\prime}(t), x^{\prime \prime}(t)\right) \in C \text { for all } t \in[a, b] .
$$

Proof. The proof follows similar ideas to that of the proof of Theorem 1 and so is only summarized. Consider the pair $\left(C^{2}([a, b]), d\right)$ where now the constants $W_{i}$ in our $d$ in (2.4) are chosen to form

$$
d(x, y):=\max \left\{\frac{\beta_{1}}{\beta_{0}} d_{\infty}(x, y), d_{\infty}\left(x^{\prime}, y^{\prime}\right), \frac{\beta_{1}}{\beta_{2}} d_{\infty}\left(x^{\prime \prime}, y^{\prime \prime}\right)\right\},
$$

(that is, $W_{0}=\beta_{1} / \beta_{0}, W_{1}=1$ and $W_{2}=\beta_{1} / \beta_{2}$ ). For the constant $R>0$ in the definition of $C$, consider the following ball $\mathscr{C}_{R} \subset C^{2}([a, b])$ defined via

$$
\mathscr{C}_{R}:=\left\{x \in C^{2}([a, b]): d(x, \phi) \leq R\right\} .
$$


Since $\mathscr{C}_{R}$ is a closed subspace of $C^{2}([a, b])$, the pair $\left(\mathscr{C}_{R}, d\right)$ forms a complete metric space.

Following the same type of arguments as in the proof of Theorem 1, for all $x \in \mathscr{C}_{R}$ we have

$$
\begin{aligned}
d(T x, \phi) & =\max \left\{\frac{\beta_{1}}{\beta_{0}} d_{\infty}(x, y), d_{\infty}\left(x^{\prime}, y^{\prime}\right), \frac{\beta_{1}}{\beta_{2}} d_{\infty}\left(x^{\prime \prime}, y^{\prime \prime}\right)\right\} \\
& \leq \max \left\{\frac{\beta_{1}}{\beta_{0}} M \beta_{0}, M \beta_{1}, \frac{\beta_{1}}{\beta_{2}} M \beta_{2}\right\} \\
& =M \beta_{1} \\
& \leq R
\end{aligned}
$$

where the final inequality holds by assumption. Thus, for all $x \in \mathscr{C}_{R}$ we have $T x \in \mathscr{C}_{R}$ so that $T: \mathscr{C}_{R} \rightarrow \mathscr{C}_{R}$. Furthermore, (4.7) and (4.8) guarantee that $T$ is contractive on $\mathscr{C}_{R}$.

The existence and uniqueness now follows from Theorem 1.

Via similar arguments, we have the following result.

Theorem 3. Let $f: D \rightarrow \mathbb{R}$ be continuous and uniformly bounded by $M>0$ on the "block"

$$
\begin{array}{r}
D:=\left\{(t, u, v, w) \in \mathbb{R}^{4}: t \in[a, b],\left|u-\frac{\lambda(t-a)^{2}}{2((b-a)-\theta(\eta-a))}\right| \leq \frac{\beta_{0}}{\beta_{2}} R,\right. \\
\left.\left|v-\frac{\lambda(t-a)}{(b-a)-\theta(\eta-a)}\right| \leq \frac{\beta_{1}}{\beta_{2}} R,\left|w-\frac{\lambda}{(b-a)-\theta(\eta-a)}\right| \leq R\right\},
\end{array}
$$

where $R>0$ is a constant and each $\beta_{i}$ is defined in (4.1) with $M \beta_{2} \leq R$. For $i=0,1,2$, let $L_{i}$ be non-negative constants (not all zero) such that

$$
\begin{array}{r}
\left|f\left(t, u_{0}, u_{1}, u_{2}\right)-f\left(t, v_{0}, v_{1}, v_{2}\right)\right| \leq \sum_{i=0}^{2} L_{i}\left|u_{i}-v_{i}\right|, \\
\text { for all }\left(t, u_{0}, u_{1}, u_{2}\right),\left(t, v_{0}, v_{1}, v_{2}\right) \in D .
\end{array}
$$

If

$$
L_{0} \beta_{0}+L_{1} \beta_{1}+L_{2} \beta_{2}<1
$$

then the BVP (1.1), (1.2) has a unique solution in $C^{3}([a, b])$ such that

$$
\left(t, x(t), x^{\prime}(t), x^{\prime \prime}(t)\right) \in D \text { for all } t \in[a, b] .
$$

Proof. Once again, the proof follows similar ideas to that of the proof of Theorem 1 and so we provide just an outline of the ideas. Consider the pair $\left(C^{2}([a, b]), d\right)$ where now the constants $W_{i}$ in our $d$ in (2.4) are chosen to form

$$
d(x, y):=\max \left\{\frac{\beta_{2}}{\beta_{0}} d_{\infty}(x, y), \frac{\beta_{2}}{\beta_{1}} d_{\infty}\left(x^{\prime}, y^{\prime}\right), d_{\infty}\left(x^{\prime \prime}, y^{\prime \prime}\right)\right\},
$$


(that is, $W_{0}=\beta_{2} / \beta_{0}, W_{1}=\beta_{2} / \beta_{1}$ and $W_{2}=1$ ). For the constant $R>0$ in the definition of $D$, consider the following ball $\mathscr{D}_{R} \subset C^{2}([a, b])$ defined via

$$
\mathscr{D}_{R}:=\left\{x \in C^{2}([a, b]): d(x, \phi) \leq R\right\} .
$$

Since $\mathscr{D}_{R}$ is a closed subspace of $C^{2}([a, b])$, the pair $\left(\mathscr{D}_{R}, d\right)$ forms a complete metric space.

Following the same type of arguments as in the proof of Theorem 1, for all $x \in \mathscr{D}_{R}$ we have

$$
\begin{aligned}
d(T x, \phi) & =\max \left\{\frac{\beta_{2}}{\beta_{0}} d_{\infty}(x, y), \frac{\beta_{2}}{\beta_{1}} d_{\infty}\left(x^{\prime}, y^{\prime}\right), d_{\infty}\left(x^{\prime \prime}, y^{\prime \prime}\right)\right\} \\
& \leq M \beta_{2} \\
& \leq R
\end{aligned}
$$

where the final inequality holds by assumption. Thus, for all $x \in \mathscr{D}_{R}$ we have $T x \in \mathscr{D}_{R}$ so that $T: \mathscr{D}_{R} \rightarrow \mathscr{D}_{R}$. Furthermore, (4.9) and (4.10) guarantee that $T$ is contractive on $\mathscr{D}_{R}$.

The existence and uniqueness now follows from Theorem 1.

Let us now form some results that approximate solutions to the unique solution $x$ of the BVP (1.1), (1.2) within certain sets. The ideas follow from Theorem 1 holding for the operator $T$ therein, see [13, Theorem 1.A].

Remark 4. Let the conditions of Theorem 1, Theorem 2 or Theorem 3 hold. If we recursively define a sequence of approximations $x_{n}=x_{n}(t)$ on $[a, b]$ via

$$
\begin{aligned}
& x_{0}:=0 \\
& x_{n+1}(t):=\frac{\lambda(t-a)^{2}}{2((b-a)-\theta(\eta-a))} \\
& +\int_{a}^{b}\left[G(t, s)+\frac{\theta \lambda(t-a)^{2}}{2((b-a)-\theta(\eta-a))} G_{t}(\eta, s)\right] f\left(s, x_{n}(s), x_{n}^{\prime}(s), x_{n}^{\prime \prime}(s)\right), \quad n=0,1,2, \cdots
\end{aligned}
$$

then, for each of the corresponding metrics defined in the proofs of Theorem 1, Theorem 2 and Theorem 3:

- the sequence $x_{n}$ converges to the solution $x$ of (1.1), (1.2) with respect to the d metric and the rate of convergence is given by

$$
d\left(x_{n+1}, x\right) \leq\left(L_{0} \beta_{0}+L_{1} \beta_{1}+L_{2} \beta_{2}\right) d\left(x_{n}, x\right) ;
$$

- for each $n$, an a priori estimate on the error is

$$
d\left(x_{n}, x\right) \leq \frac{\left(L_{0} \beta_{0}+L_{1} \beta_{1}+L_{2} \beta_{2}\right)^{n}}{1-\left(L_{0} \beta_{0}+L_{1} \beta_{1}+L_{2} \beta_{2}\right)} d\left(x_{1}, 0\right)
$$

- for each $n$, an a posteriori estimate on the error is

$$
d\left(x_{n+1}, x\right) \leq \frac{\left(L_{0} \beta_{0}+L_{1} \beta_{1}+L_{2} \beta_{2}\right)}{1-\left(L_{0} \beta_{0}+L_{1} \beta_{1}+L_{2} \beta_{2}\right)} d\left(x_{n+1}, x_{n}\right) .
$$




\subsection{Fixed Point Approach with Two Metrics}

Once again, so that we can avoid the repeated use of complicated expressions, let us define the following constants with the aim of simplifying certain notation. Let $p>1$ and $q>1$ be constants such that $1 / p+1 / q=1$. Define

$$
\begin{aligned}
c_{0} & :=\max _{t \in[a, b]}\left[\int_{a}^{b}\left|G(t, s)+\frac{\theta \lambda(t-a)^{2}}{2((b-a)-\theta(\eta-a))} G_{t}(\eta, s)\right|^{q} d s\right]^{1 / q} ; \\
c_{1} & :=\max _{t \in[a, b]}\left[\int_{a}^{b}\left|G_{t}(t, s)+\frac{\theta \lambda(t-a)}{(b-a)-\theta(\eta-a)} G_{t}(\eta, s)\right|^{q} d s\right]^{1 / q} ; \\
c_{2} & :=\max _{t \in[a, b]}\left[\int_{a}^{b}\left|G_{t t}(t, s)+\frac{\theta \lambda}{(b-a)-\theta(\eta-a)} G_{t}(\eta, s)\right|^{q} d s\right]^{1 / q} ;
\end{aligned}
$$

and

$$
\begin{aligned}
\gamma_{0} & :=\left[\int_{a}^{b}\left[\int_{a}^{b}\left|G(t, s)+\frac{\theta \lambda(t-a)^{2}}{2((b-a)-\theta(\eta-a))} G_{t}(\eta, s)\right|^{p} d s\right]^{p / q}\right]^{1 / p} \\
\gamma_{1} & :=\left[\int_{a}^{b}\left[\int_{a}^{b}\left|G_{t}(t, s)+\frac{\theta \lambda(t-a)}{((b-a)-\theta(\eta-a))} G_{t}(\eta, s)\right|^{p} d s\right]^{p / q}\right]^{1 / p} \\
\gamma_{2} & :=\left[\int_{a}^{b}\left[\int_{a}^{b}\left|G_{t t}(t, s)+\frac{\theta \lambda}{((b-a)-\theta(\eta-a))} G_{t}(\eta, s)\right|^{p} d s\right]^{p / q}\right]^{1 / p} .
\end{aligned}
$$

We will draw on the following relationship between the two metrics $\delta$ and $d$ in the proof of our main result.

Theorem 5. For $x, y \in C^{2}([a, b])$ we have

$$
\delta(x, y) \leq(b-a)^{1 / p}\left(\frac{L_{0}}{W_{0}}+\frac{L_{1}}{W_{1}}+\frac{L_{2}}{W_{2}}\right) d(x, y) .
$$

Proof. See [1, Theorem 13] for a proof.

Let us now state and prove our results on existence and uniqueness of solutions to (1.1), (1.2) where we employ two metrics under Rus's theorem.

Theorem 6. Let $f: B \rightarrow \mathbb{R}$ be continuous and uniformly bounded by $M>0$ on the "block" $B$ defined in Theorem 1 with $M \beta_{0} \leq R$. For $i=0,1,2$, let $L_{i}$ be non-negative constants (not all zero) such that

$$
\begin{array}{r}
\left|f\left(t, u_{0}, u_{1}, u_{2}\right)-f\left(t, v_{0}, v_{1}, v_{2}\right)\right| \leq \sum_{i=0}^{2} L_{i}\left|u_{i}-v_{i}\right|, \\
\text { for all }\left(t, u_{0}, u_{1}, u_{2}\right),\left(t, v_{0}, v_{1}, v_{2}\right) \in B .
\end{array}
$$


If there are constants $p>1$ and $q>1$ with $1 / p+1 / q=1$ such that

$$
L_{0} \gamma_{0}+L_{1} \gamma_{1}+L_{2} \gamma_{2}<1
$$

where each of the $\gamma_{i}$ are defined in (4.12), then the BVP (1.1), (1.2) has a unique solution in $C^{3}([a, b])$ such that

$$
\left(t, x(t), x^{\prime}(t), x^{\prime \prime}(t)\right) \in B \text { for all } t \in[a, b]
$$

Proof. Define $\mathscr{B}_{R}, d$ and $T$ as in the proof of Theorem 1. We want to show that there exists a unique $x \in \mathscr{B}_{R}$ such that

$$
T x=x .
$$

Such a solution will also lie in $C^{3}([a, b])$ as can be directly shown by differentiating (3.1) and confirming the continuity.

To establish the existence and uniqueness to $T x=x$, we show that the conditions of Theorem 2 hold.

The pair $\left(\mathscr{B}_{R}, d\right)$ forms a complete metric space. Following the same type of arguments as in the proof of Theorem 1 it can be shown that the condition $M \beta_{0} \leq R$, ensures $T: \mathscr{B}_{R} \rightarrow \mathscr{B}_{R}$.

In addition, consider the metric $\delta$ in (2.5) on $\mathscr{B}_{R}$ where $p>1$ and the $L_{i}$ come from (4.14).

For $x, y \in \mathscr{B}_{R}$ and $t \in[a, b]$, consider

$$
\begin{aligned}
& |(T x)(t)-(T y)(t)| \\
\leq & \int_{a}^{b}\left|G(t, s)+\frac{\theta \lambda(t-a)^{2}}{2((b-a)-\theta(\eta-a))} G_{t}(\eta, s)\right|\left(\sum_{i=0}^{2} L_{i}\left|x^{(i)}(s)-y^{(i)}(s)\right|\right) d s \\
\leq & {\left[\int_{a}^{b}\left|G(t, s)+\frac{\theta \lambda(t-a)^{2}}{2((b-a)-\theta(\eta-a))} G_{t}(\eta, s)\right|^{q} d s\right]^{1 / q} } \\
& \times\left(\sum_{i=0}^{2} L_{i}\left(\int_{a}^{b}|x(s)-y(s)|^{p} d s\right)^{1 / p}\right) \\
= & c_{0} \delta(x, y) .
\end{aligned}
$$

Above, we have used (4.14) and Hoelder's inequality [4,8] to obtain (4.16). Similar calculations lead us to

$$
\begin{aligned}
\left|(T x)^{\prime}(t)-(T y)^{\prime}(t)\right| & \leq c_{1} \delta(x, y) \\
\left|(T x)^{\prime \prime}(t)-(T y)^{\prime \prime}(t)\right| & \leq c_{2} \delta(x, y) .
\end{aligned}
$$

Combining the above inequalities we obtain

$$
d(T x, T y) \leq c \delta(x, y), \text { for some } c>0 \text { and all } x, y \in \mathscr{B}_{R},
$$

where

$$
c:=\max \left\{c_{0}, \frac{\beta_{0}}{\beta_{1}} c_{1}, \frac{\beta_{0}}{\beta_{2}} c_{2}\right\} .
$$


Thus, the inequality (2.2) of Theorem 2 holds.

Furthermore, $T$ is continuous on $\mathscr{B}_{R}$ with respect to the $d$ metric as can be shown from the following arguments. For all $x, y \in \mathscr{B}_{R}$ we may apply (4.13) from Theorem 5 to (4.17) to obtain

$$
\begin{aligned}
d(T x, T y) & \leq c \delta(x, y) \\
& \leq c(b-a)^{1 / p}\left(L_{0}+L_{1} \frac{\beta_{1}}{\beta_{0}}+L_{2} \frac{\beta_{2}}{\beta_{0}}\right) d(x, y) .
\end{aligned}
$$

Thus, given any $\varepsilon>0$ we can choose

$$
\Delta=\frac{\varepsilon}{c(b-a)^{1 / p}\left(L_{0}+L_{1} \frac{\beta_{1}}{\beta_{0}}+L_{2} \frac{\beta_{2}}{\beta_{0}}\right)},
$$

so that $d(T x, T y)<\varepsilon$ whenever $d(x, y)<\Delta$. Hence $T$ is continuous on $\mathscr{B}_{R}$ with respect to the $d$ metric.

Finally, we show that $T$ is contractive on $\mathscr{B}_{R}$ with respect to the $\delta$ metric, that is, the inequality (2.3) in Theorem 2 holds. From (4.16) and the associated discussion, for each $x, y \in \mathscr{B}_{R}$ and $t \in[a, b]$ we have

$$
\begin{gathered}
\left(\int_{a}^{b}|(T x)(t)-(T y)(t)|^{p} d t\right)^{1 / p} \leq \gamma_{0} \delta(x, y) ; \\
\left(\int_{a}^{b}\left|(T x)^{\prime}(t)-(T y)^{\prime}(t)\right|^{p} d t\right)^{1 / p} \leq \gamma_{1} \delta(x, y) ; \\
\left(\int_{a}^{b}\left|(T x)^{\prime \prime}(t)-(T y)^{\prime \prime}(t)\right|^{p} d t\right)^{1 / p} \leq \gamma_{2} \delta(x, y) ;
\end{gathered}
$$

and so we obtain

$$
\delta(T x, T y) \leq\left(L_{0} \gamma_{0}+L_{1} \gamma_{1}+L_{2} \gamma_{2}\right) \delta(x, y) .
$$

From our assumption (4.15), we thus have

$$
\delta(T x, T y) \leq \alpha \delta(x, y)
$$

for some $\alpha<1$ and all $x, y \in \mathscr{B}_{R}$.

Thus, Theorem 2 is applicable and the operator $T$ has a unique fixed point in $\mathscr{B}_{R}$. This solution is also in $C^{3}([a, b])$ and we have equivalently shown that the BVP $(1.1),(1.2)$ has a unique solution.

Similarly, we have the following two results which we state without proof due to concerns of brevity and repetition. The proofs follow similar lines to that of the proof of Theorem 6 . 
Theorem 7. Let $f: C \rightarrow \mathbb{R}$ be continuous and uniformly bounded by $M>0$ on the "block" $C$ defined in Theorem 2 with $M \beta_{1} \leq R$. For $i=0,1,2$, let $L_{i}$ be non-negative constants (not all zero) such that

$$
\begin{array}{r}
\left|f\left(t, u_{0}, u_{1}, u_{2}\right)-f\left(t, v_{0}, v_{1}, v_{2}\right)\right| \leq \sum_{i=0}^{2} L_{i}\left|u_{i}-v_{i}\right|, \\
\text { for all }\left(t, u_{0}, u_{1}, u_{2}\right),\left(t, v_{0}, v_{1}, v_{2}\right) \in C .
\end{array}
$$

If there are constants $p>1$ and $q>1$ with $1 / p+1 / q=1$ such that

$$
L_{0} \gamma_{0}+L_{1} \gamma_{1}+L_{2} \gamma_{2}<1
$$

where each of the $\gamma_{i}$ are defined in (4.12), then the BVP (1.1), (1.2) has a unique solution in $C^{3}([a, b])$ such that

$$
\left(t, x(t), x^{\prime}(t), x^{\prime \prime}(t)\right) \in C \text { for all } t \in[a, b]
$$

Theorem 8. Let $f: D \rightarrow \mathbb{R}$ be continuous and uniformly bounded by $M>0$ on the "block" $D$ defined in Theorem 3 with $M \beta_{2} \leq R$. For $i=0,1,2$, let $L_{i}$ be non-negative constants (not all zero) such that

$$
\begin{array}{r}
\left|f\left(t, u_{0}, u_{1}, u_{2}\right)-f\left(t, v_{0}, v_{1}, v_{2}\right)\right| \leq \sum_{i=0}^{2} L_{i}\left|u_{i}-v_{i}\right|, \\
\text { for all }\left(t, u_{0}, u_{1}, u_{2}\right),\left(t, v_{0}, v_{1}, v_{2}\right) \in D .
\end{array}
$$

If there are constants $p>1$ and $q>1$ with $1 / p+1 / q=1$ such that

$$
L_{0} \gamma_{0}+L_{1} \gamma_{1}+L_{2} \gamma_{2}<1
$$

where each of the $\gamma_{i}$ are defined in (4.12), then the BVP (1.1), (1.2) has a unique (nontrivial) solution in $C^{3}([0,1])$ such that

$$
\left(t, x(t), x^{\prime}(t), x^{\prime \prime}(t)\right) \in D \text { for all } t \in[a, b] .
$$

Remark 9. Let $m:=d_{\infty}\left(f\left(\cdot, \phi, \phi^{\prime}, \phi^{\prime \prime}\right), 0\right)$. Each of the invariance conditions can be replaced with

$$
m \beta_{i} \leq\left(1-\left(L_{0} \beta_{0}+L_{1} \beta_{1}+L_{2} \beta_{2}\right)\right) R
$$

in our existence theorems herein and their conclusions will still hold. To see this, for example, we show that $T: \mathscr{B}_{R} \rightarrow \mathscr{B}_{R}$. For all $x \in \mathscr{B}_{R}$ we have

$$
\begin{aligned}
d(T x, \phi) & \leq d(T x, T \phi)+d(T \phi, \phi) \\
& \leq\left(L_{0} \beta_{0}+L_{1} \beta_{1}+L_{2} \beta_{2}\right) d(x, \phi)+m \beta_{0} \\
& \leq\left(L_{0} \beta_{0}+L_{1} \beta_{1}+L_{2} \beta_{2}\right) R+\left(1-\left(L_{0} \beta_{0}+L_{1} \beta_{1}+L_{2} \beta_{2}\right)\right) R \\
& =R .
\end{aligned}
$$


Thus we see that under this condition we ensure that $T: \mathscr{B}_{R} \rightarrow \mathscr{B}_{R}$. The other cases may be shown in similar ways.

\section{EXAMPLE AND REMARK}

Let us probe an example to show how our new results can be applied to concrete problems.

Example 5.1. The nonlinear differential equation (1.3) subject to

$$
x(0)=0, x^{\prime}(0)=0, x^{\prime}(1)=1
$$

has a unique solution such that

$$
\left(t, x(t), x^{\prime}(t), x^{\prime \prime}(t)\right) \in B, \text { for all } t \in[0,1]
$$

where

$$
B:=\left\{(t, u, v, w) \in \mathbb{R}^{4}: t \in[a, b],\left|u-\frac{t^{2}}{2}\right| \leq \frac{1}{2},\left|v-\frac{t}{2}\right| \leq \frac{3}{4},\left|w-\frac{1}{2}\right| \leq 6\right\} .
$$

Proof. We show that the conditions of Theorem 1 are satisfied with $a=0, \lambda=1, \theta=0$ and $R=1 / 2$ is to be fixed a little later.

We have

$$
\frac{\beta_{1}}{\beta_{0}}=\frac{3}{2} ; \quad \frac{\beta_{2}}{\beta_{0}}=6
$$

Also, on $[0,1]$, the function $\phi$ from the proof of Theorem 1 satisfies

$$
|\phi| \leq 1 / 2 ;\left|\phi^{\prime}\right| \leq 1 ;\left|\phi^{\prime \prime}\right| \leq 1
$$

Now, the partial derivatives of $f(t, u, v, w):=u w$ are continuous and uniformly bounded on $B$, namely

$$
\begin{aligned}
\left|f_{u}\right| & =|w| \leq\left|w-\phi^{\prime \prime}\right|+\left|\phi^{\prime \prime}\right| \leq 6 R+1 \\
\left|f_{w}\right| & =|u| \leq|u-\phi|+|\phi| \leq R+1 / 2 .
\end{aligned}
$$

The value of these bounds can be used as our Lipschitz constants:

$$
L_{0}=6 R+1 ; \quad L_{1}=0 ; \quad L_{2}=R+1 / 2
$$

The bound $M$ on our $f$ can be calculated via

$$
\begin{aligned}
|f(t, u, v, w)| & =|u w| \\
& =|(u-\phi+\phi)|\left|\left(w-\phi^{\prime \prime}+\phi^{\prime \prime}\right)\right| \\
& \leq(R+1 / 2)(6 R+1) .
\end{aligned}
$$


Thus

$$
M \beta_{0}=(R+1 / 2)(6 R+1) / 12
$$

and

$$
L_{0} \beta_{0}+L_{1} \beta_{1}+L_{2} \beta_{2}=(6 R+1) / 12+0+(R+1 / 2) / 2=R+1 / 3 .
$$

Now, we see that for the choice $R=1 / 2$ we have $M \beta_{0} \leq R$ and $L_{0} \beta_{0}+L_{1} \beta_{1}+L_{2} \beta_{2}<1$.

Thus, we see that all of the conditions of Theorem 1 are satisfied and its conclusion holds for this example.

Remark 1. We remark that the contraction condition (4.15) can be applied to problems where (4.3) does not necessarily apply and it is not difficult to construct a $f$ to illustrate this (see, for example, [2]).

\section{REFERENCES}

[1] S.S. Almuthaybiri \& C.C. Tisdell. Existence and uniqueness of solutions to third-order boundary value problems: analysis in closed and bounded sets. Differ. Equ. Appl., 12(12) (2020), 291-312. doi:10.7153/dea-2020-12-19.

[2] S.S. Almuthaybiri \& C.C. Tisdell. Sharper Existence and Uniqueness Results for Solutions to ThirdOrder Boundary Value Problems. Math. Model. Anal., 25(3) (2020), 409-420. doi:10.3846/mma.2020. 11043.

[3] S. Banach. Sur les opérations dans les ensembles abstraits et leur application aux équations intégrales. Fund. Math., 3 (1922), 133-181.

[4] O. Hoelder. Ueber einen Mittelwertsatz (German). J Goett. Nachr., (1889), 38-47.

[5] A.L.M. Martinez, M.R.A. Ferreira \& E.V. Castelani. Theoretical and numerical aspects of a thirdorder three-point nonhomogeneous boundary value problem. TEMA Tend. Mat. Apl. Comput., 20(3) (2019), 417-427. doi:10.5540/tema.2019.020.03.417.

[6] J. Merkin. On dual solutions occurring in mixed convection in a porous medium. Journal of Engineering Mathematics, 20 (1986), 171-179. doi:10.1007/BF00042775.

[7] J.H. Merkin. Mixed convection boundary layer flow on a vertical surface in a saturated porous medium. J. Engrg. Math., 14(4) (1980), 301-313. doi:10.1007/BF00052913.

[8] L.J. Rogers. An extension of a certain theorem in inequalities. Messenger of Math., 17 (1888), 145150.

[9] H. Rouse. "Advanced mechanics of fluids", volume 12. R. E. Krieger Pub. Co, Huntington, N.Y (1976), 444 pp.

[10] I.A. Rus. On a fixed point theorem of Maia. Studia Univ. Babeş-Bolyai Math., 22 (1977), 40-42.

[11] S. Smirnov. Green's function and existence of a unique solution for a third-order three-point boundary value problem. Math. Model. Anal., 24(2) (2019), 171-178. doi:10.3846/mma.2019.012. 
[12] C.C. Tisdell. On Picard's iteration method to solve differential equations and a pedagogical space for otherness. Internat. J. Math. Ed. Sci. Tech., 50(5) (2019), 788-799. doi:10.1080/0020739X.2018. 1507051.

[13] E. Zeidler. "Nonlinear functional analysis and its applications. I". Springer-Verlag, New York (1986), xxi+897 pp. doi:10.1007/978-1-4612-4838-5. Fixed-point theorems, Translated from the German by Peter R. Wadsack.

(cc) BY 